\title{
Kritisches Denken im naturwissenschaftlichen Unterricht - Synergiemodell, Problemlage und Desiderata
}

\author{
Susanne Rafolt ${ }^{1}$ (D) Suzanne Kapelari ${ }^{1}$ (D) Kerstin Kremer $^{2,3}$ (D) \\ Eingegangen: 31. Mai 2018 / Angenommen: 10. Mai 2019/Online publiziert: 23. Mai 2019 \\ (c) Der/die Autor(en) 2019
}

\section{Zusammenfassung}

Kritisches Denken (KD) gilt weitläufig als ein fundamentales Bildungsziel. In naturwissenschaftsdidaktischer Literatur wird diskutiert, dass KD einen Rahmen für die Auseinandersetzung mit den Konzepten Socio-Scientific Issues und Nature of Science bildet. Dennoch fehlt in der Naturwissenschaftsdidaktik ein einvernehmliches Verständnis des Konstrukts KD, was die Weiterentwicklung des Forschungsgebietes und die Implementierung in den naturwissenschaftlichen Unterricht erschwert. Das hier vorgestellte theoriegeleitete Synergiemodell des kritischen Denkens fasst den abstrakten wissenschaftlichen Diskurs für die naturwissenschaftsdidaktische Forschung und die Unterrichtsentwicklung zusammen. Es schafft sowohl eine Grundlage für die Analyse von Unterrichtsprozessen als auch eine Diskussionsbasis für die Verständigung über die Charakteristika von KD im Kontext von naturwissenschaftlicher Bildung und gesellschaftlichen Herausforderungen. Aufbauend auf der theoretischen Klärung werden Desiderata für die fachspezifische Konzeptualisierung sowie die explizite Implementierung des Konstrukts KD in den naturwissenschaftlichen Unterricht und die Aus- und Weiterbildung von Lehrkräften naturwissenschaftlicher Fächer abgeleitet. Ziel dieses Artikels ist es, einen wissenschaftlichen sowie einen davon ausgehenden praktischen Diskurs darüber anzuregen, welche Charakteristika KD im naturwissenschaftlichen Unterricht auszeichnen.

Schlüsselwörter Kritik · Nature of Science $\cdot$ Socio-Scientific Issues (SSI) · Wissen · Kompetenz · Bildungsziel

Susanne Rafolt

susanne.rafolt@uibk.ac.at

1 Institut für Fachdidaktik, Bereich Didaktik der

Naturwissenschaften, Geographie, Informatik

und Mathematik, Universität Innsbruck,

Technikerstraße 25, 6020 Innsbruck, Österreich

2 IDN - Institut für Didaktik der Naturwissenschaften,

Fachgebiet Didaktik der Biologie, Leibniz Universität

Hannover, Am Kleinen Felde 30, 30168 Hannover,

Deutschland

3 IPN - Leibniz-Institut für die Pädagogik der

Naturwissenschaften und Mathematik, Abteilung Didaktik der Biologie, Christian-Albrechts-Universität zu Kiel, Kiel, Deutschland 


\title{
Critical Thinking in the Science Classroom—Synergy Model, Challenges and Desiderata
}

\begin{abstract}
Critical Thinking is widely considered a fundamental educational goal. In science education research, critical thinking is discussed as a framework for dealing with the concepts socio-scientific issues and nature of science. However, a lack of consensus regarding the construct of critical thinking complicates developing the research field and implementing critical thinking in science education. The proposed theory-led Synergy Model of Critical Thinking summarises the complex scientific discourse for teaching practice and science education research. It provides a basis for the analysis of teaching processes and stimulates discussions about the characteristics of critical thinking in the context of science education and socio-scientific issues. Based on the theoretical clarification, we derive desiderata for the subject-specific conceptualisation of the construct critical thinking as well its explicit implementation in science teaching and teacher education. This article aims at clarifying critical thinking and stimulating both a scientific and practical discourse on the characteristics of critical thinking in science education.
\end{abstract}

Keywords Critique $\cdot$ Nature of Science $\cdot$ Socio-scientific Issues (SSI) $\cdot$ Knowledge $\cdot$ Skill $\cdot$ Educational goal

\section{Einleitung}

In der internationalen naturwissenschaftsdidaktischen Literatur werden komplexe gesellschaftliche Herausforderungen ohne eindeutigen Lösungsweg, die auch naturwissenschaftliche Aspekte beinhalten oder ein naturwissenschaftliches Verständnis erfordern, unter dem Begriff Socio-Scientific Issues (SSI) zusammengefasst und als Bezugsrahmen für naturwissenschaftlichen Unterricht intensiv diskutiert (Zeidler 2014). Um sich mit SSI fundiert auseinandersetzen zu können, sollen im Sinne des internationalen naturwissenschaftlichen Bildungsdiskurses neben einer naturwissenschaftlichen Grundbildung (Scientific Literacy) auch ein Verständnis von den Charakteristika der Naturwissenschaften (Nature of Science) und ein kritisches Denken gefördert werden (Bybee 1997; Gunn et al. 2008; Kolstø et al. 2006; Osborne 2014; Yacoubian 2015; Zeidler 2014).

Kritisches Denken (KD) ist ein zentrales Thema der internationalen Forschung in den Bereichen Erziehungsund Bildungswissenschaft (z. B. Bailin et al. 1999b; Pithers und Soden 2000; Winch 2003), Naturwissenschaftsdidaktik (z.B. Bailin 2002; Gunn et al. 2008; Vieira et al. 2011; Yacoubian 2015), Philosophie (z. B. Lipman 1988; Paul und Elder 2014) und Psychologie (z. B. Dwyer et al. 2014; Greene und Yu 2016; Halpern 2014; Kuhn 1999; Schraw et al. 2006; Sinatra et al. 2014; Stanovich 2016). Dabei bleibt KD in Hinblick auf die Förderung meist undifferenziert und anwendbare sowie empirisch geprüfte Umsetzungsvorschläge vor allem für den Fachunterricht in den Naturwissenschaften fehlen. Zudem ist KD als Konstrukt aus der Perspektive der Fachdidaktiken kaum erforscht. Es bleibt bisher weitestgehend unbeantwortet, wie ein elaboriertes konzeptuelles Verständnis von KD als ein fundamentales Werkzeug für die Auseinandersetzung mit naturwissenschaftlichem Wissen und gesellschaftlichen Herausforderungen vermittelt und evaluiert werden kann.
Dieser Artikel führt in den vielschichtigen und interdisziplinären wissenschaftlichen Diskurs über KD ein. Zunächst wird die Problematik einer Definition des Konstrukts diskutiert. Dabei wird auch auf den philosophisch umstrittenen Kritikbegriff eingegangen und erläutert, inwiefern sich dieser als Substantiv von der Nominalphrase KD unterscheidet. In Hinblick auf die kritische Auseinandersetzung mit SSI im naturwissenschaftlichen Unterricht wird ein theoriegeleitetes Synergiemodell des kritischen Denkens vorgestellt. Dieses Modell übernimmt eine wichtige Vermittlerfunktion und dient dazu, die wesentlichen Facetten idealtypischen $\mathrm{KD}$ miteinander in Beziehung zu setzen, zu visualisieren und so für den wissenschaftlichen Diskurs und die unterrichtliche Praxis zu veranschaulichen. Es folgt eine Diskussion zu KD als Bildungsziel und in der Wissensgesellschaft. Dabei werden Bezüge zur Betrachtung von SSI und von $\mathrm{Na}$ ture of Science hergestellt. Abschließend werden aufbauend auf der theoretischen Klärung Desiderata für die Reflexion von Möglichkeiten und Grenzen des Konstrukts KD sowie die sensible Implementierung in den naturwissenschaftlichen Unterricht, die naturwissenschaftliche Lehrkräftebildung und die naturwissenschaftsdidaktische Forschung abgeleitet. Hiermit verbunden ist eine Verknüpfung zu den jeweiligen Unterrichtsfächern herzustellen, um die Umsetzung im Unterricht sowie in der Unterrichtsforschung und in der Lehrkräftebildung zu erleichtern. Ferner wird die fehlende Konzeptualisierung und Operationalisierung von KD diskutiert.

\section{Problematik der Definition kritischen Denkens}

Anfang der Neunziger Jahre des letzten Jahrhunderts arbeiteten Facione und Kollegen, darunter auch Ennis, Lipman und Paul, an einem fachunabhängigen konsensorien- 
tierten Verständnis von KD und einer sich daraus ableitenden idealtypischen Beschreibung eines kritischen Denkers bzw. einer kritischen Denkerin (Facione 1990). Dieser Konsens beschreibt KD sehr umfassend. Das in diesem Artikel präsentierte Synergiemodell des kritischen Denkens (vgl. Abschn. 3) greift das Verständnis von Facione und Kollegen aus dem Jahr 1990 auf, erweitert es und erklärt es textlich wie auch visuell. Denn in Hinblick auf die darauffolgenden wissenschaftlichen Publikationen scheint dieser Konsens nicht die notwendige Klärung erreicht zu haben (Bailin et al. 1999a; McMillan 1987; Winch 2003; Zeidler et al. 1992). In vielen Publikationen wird KD ohne Erklärung aufgegriffen (z. B. Klosterman und Sadler 2010; Sinatra et al. 2014), sehr vereinfachend mit Problemlösung beschrieben, fälschlicherweise mit der Bloom'schen Taxonomie gleichgesetzt oder als ein Sammelbegriff für alle möglichen Denkoperationen verwendet (Zeidler et al. 1992). Dabei ist nicht geklärt, ob und inwiefern KD evaluiert und von anderen Arten des Denkens, wie dem rationalen Denken, klar abgegrenzt werden kann (Astleitner 1998; Kennedy et al. 1990; Stanovich 2016) und unterschiedliche Disziplinen dasselbe darunter verstehen (Bailin 2002; Davies 2013; Kennedy et al. 1990; Moore 2013). Dies erschwert die Weiterentwicklung des Forschungsgebietes (Astleitner 1998; Bailin et al. 1999a; McMillan 1987; Winch 2003; Zeidler et al. 1992).

Die Uneinigkeit über die Bedeutung, Entwicklung, Förderung und Evaluierung von KD fußt nicht nur auf der geringen Greifbarkeit dessen, was Denken ist, sondern auch auf der unklaren Bedeutung von Kritik, für die „es keine fest umrissenen Grenzen“ gibt (Winch 2003, S. 30). Andererseits ist das Konstrukt KD nicht gleichbedeutend mit dem der Kritik. In manchen Publikationen wird jedoch das Konstrukt KD mit anderen Nominalphrasen vermischt (z. B. OECD 2018; Osborne 2014; Winch 2003). Beispielsweise verwendet die Europäische Union (2006) explizit den Begriff KD $(5,7)$ und setzt diesen anschließend damit gleich, kritisch zu sein und etwas kritisch zu tun. Im Kontext dieses Artikels bedarf es daher einer Klärung des Kritikbegriffes und dessen Verwendung als Nominalphrase (KD). Während KD im anschließenden Abschnitt ausführlich erklärt wird (s. Synergiemodell des kritischen Denkens), erfolgt hier ein Einblick in die nicht klar umrissene und philosophisch umstrittene Bedeutung des Kritikbegriffes (Röttgers 1975; Jaeggi und Wesche 2009; Winch 2003).

Kritik kann als ,eine allgemein prüfende Einstellung gegenüber selbstverständlichen Wissensbeständen in allen Bereichen menschlicher Erfahrung" (Lembeck 1996, S. 279) interpretiert werden. ,Die Frage nach den Bedingungen und der Möglichkeit von Kritik stellt sich immer dort, wo Gegebenheiten analysiert, beurteilt oder als falsch abgelehnt werden" (Jaeggi und Wesche 2009, S. 7). Sie ist außerdem eng mit ideologischen Positionen und gesellschaftli- chen Veränderungen verknüpft (Röttgers 1975; Jaeggi und Wesche 2009; Winch 2003). Der Kritikbegriff leitet sich vom altgriechischen Verb krínein ab, das trennen, sichten, auswählen und beurteilen bedeutet, und ist mit den Begriffen Krise und Kriterium verwandt (Bittner 2009; Röttgers 1975; Schneiders 1985). Im Sinne ihres etymologischen Ursprungs kann Kritik als die regelgeleitete Auseinandersetzung mit zuvor gesonderten Faktoren interpretiert werden, beispielsweise als Gegenüberstellung von negativen und positiven Aspekten, um so zu einem Urteil oder einer Bewertung zu kommen (Bittner 2009). Die Bezugspunkte der Kritik und die Regeln, nach denen die Auseinandersetzung erfolgt, haben sich jedoch mit den gesellschaftlichen Entwicklungen der Epochen gewandelt. Während sich die Kritik lange auf medizinische oder juristische Bereiche bezog, wie ein Krankheitszustand oder Rechtsfall, gerieten mit der Aufklärung auch (Bibel-)Texte, Kunstwerke und soziale Verhältnisse in den Fokus von Kritik. Seither kam dem Zweifel eine stärker werdende Rolle zu und der Kritikbegriff wurde zunehmend auch negativ konnotiert (Halpern 2014; Röttgers 1975; Schneiders 1985). Durch die Betrachtung ihrer etymologischen Wurzeln wird deutlich, dass Kritik nicht automatisch in der Ablehnung des kritisierten Objekts endet. Kritik „,bedeutet immer gleichzeitig Dissoziation wie Assoziation. Sie unterscheidet, trennt und distanziert sich; und sie verbindet, setzt in Beziehung, stellt Zusammenhänge her" (Jaeggi und Wesche 2009, S. 8).

\section{Synergiemodell des kritischen Denkens}

Das Synergiemodell des kritischen Denkens (Abb. 1) beschreibt KD als eine Synergie von Charakteristika, die nicht logisch, psychologisch oder epistemologisch geordnet sind, keiner Hierarchie unterliegen (Facione 1990) und nicht unabhängig voneinander wirken, sondern ineinandergreifen. Es fasst den abstrakten, wissenschaftlichen Diskurs für die unterrichtliche Praxis und die naturwissenschaftsdidaktische Forschung zusammen und beruht auf der Zusammenschau internationaler wissenschaftlicher Literatur, die sich mit Charakteristika sowie Möglichkeiten und Grenzen von KD auseinandersetzt. In der Beschreibung der Abbildung können nicht alle Autorinnen und Autoren genannt werden, daher dienen die Quellenverweise im Text der Orientierung in der weiterführenden Literatur.

Vor dem Hintergrund des vorausgegangenen Abschnitts verstehen wir KD als ein Konstrukt, das in seiner Komplexität und Vielschichtigkeit im Synergiemodell des kritischen Denkens dargestellt wird. Es übernimmt eine Vermittlerfunktion, indem es die wesentlichen Facetten idealtypischen KD miteinander in Beziehung setzt und für den wissenschaftlichen Diskurs sowie die unterrichtliche Praxis veranschaulicht. So dient das Synergiemodell des kritischen 
Abb. 1 Synergiemodell des kritischen Denkens
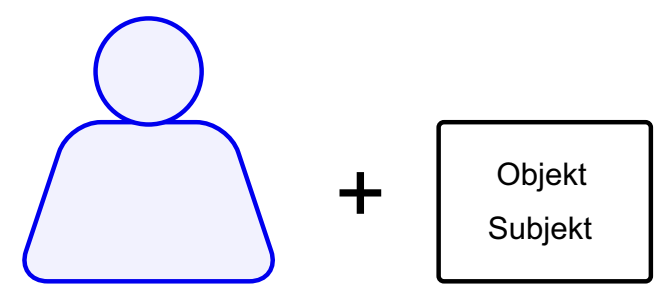

Wissen

Fähigkeiten

Haltung und Motivation

Normen,Werte und Emotionen
Intellektuelle Standards

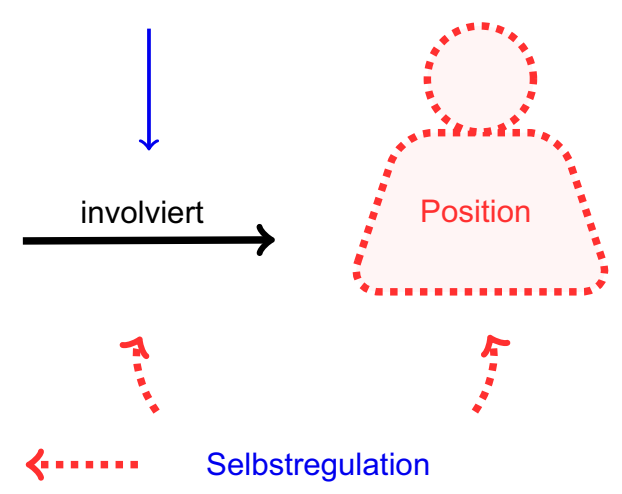

Denkens auch als Grundlage für die qualitative Analyse von Unterrichtsprozessen sowie der Verständigung über die Charakteristika von KD im Kontext von naturwissenschaftlichem Unterricht und in der Aus- und Weiterbildung naturwissenschaftlicher Lehrkräfte. Ferner stellt es einen Referenzrahmen für die Auseinandersetzung mit Socio-Scientific Issues und den dafür notwendigen Bedingungen (vgl. Abschn. 5) dar.

Im Synergiemodell des kritischen Denkens werden Naturwissenschaften als kognitiv-epistemisches und als sozial-institutionelles System betrachtet (Erduran und Dagher 2014; vgl. Abschn. 5). Kontextunabhängig ist es an keine wissenschaftstheoretische Richtung gebunden und hat keine normative Ausrichtung. Viele Aspekte, insbesondere die Bedeutung der intellektuellen Standards und die Selbstregulation, lassen sich jedoch im Kritischen Rationalismus verorten (vgl. Popper 1984).

Ob und in welcher Qualität KD in den Unterricht bzw. in die Lehrkräftebildung einfließt und somit Schülerinnen und Schülern sowie Lehrkräften vermittelt werden kann, hängt auch davon ab, ob Lehrende eine klare Vorstellung davon haben, was KD ist und wie es beobachtet werden kann (Ab Kadir 2017; Bailin et al. 1999b; Lipman 1988). Das Synergiemodell des kritischen Denkens schlägt eine Brücke zwischen Theorie und Praxis, indem es die Wahrnehmung und Kommunikation von Veränderungen in der Performanz der Lernenden zunächst qualitativ beschreibbar macht sowie eine Reflexion und Diskussion notwendiger Bedingungen für den Unterricht oder die Unterrichtsforschung ermöglicht.

\section{Involviertsein mit einem Objekt oder Subjekt und Positionierung}

Das Synergiemodell des kritischen Denkens zeigt das Aufeinandertreffen des kritischen Denkers bzw. der kritischen Denkerin mit einem Objekt (z.B. ein Problem, eine Frage, eine Behauptung) oder einem Subjekt (z.B. mit der eigenen Haltung, mit Personen oder Institutionen). Durch das Involviertsein mit dem Objekt oder Subjekt kommt es zu einer individuellen Positionierung oder der Veränderung einer bereits erreichten Position. Die Positionierung kann auch als Entscheidung oder Bewertung verstanden werden (Kuhn 1999; Lipman 1988). Dieser Prozess wird beim KD durch eine Synergie von Charakteristika und Kriterien geleitet, die im Folgenden vorgestellt werden. Diese sind nicht logisch, psychologisch oder epistemologisch geordnet, unterliegen keiner Hierarchie (Facione 1990, S. 11) und wirken nicht unabhängig voneinander, sondern greifen ineinander. KD kann nicht auf diskrete mentale Prozesse, Verfahrensschritte oder Teilaspekte reduziert werden und über diese abgearbeitet werden, beispielsweise indem sie unzusammenhängend geübt werden, da sich KD nur durch das Zusammenspiel all seiner Facetten erschließt (Bailin et al. 1999a, b; Ennis 1990; Halpern 2014; McMillan 1987).

\section{Intellektuelle Standards}

KD ist nicht unstrukturiert und ziellos, sondern kriteriengeleitet (Bailin et al. 1999b; Lipman 1988; Paul und Elder 2014). Intellektuelle Standards (Paul und Elder 2014) stellen sicher, dass das Involviertsein mit dem Objekt oder Subjekt korrekt, klar und präzise, relevant und signifikant, autonom, fair und neutral sowie logisch und rational ist und sowohl in die Breite als auch in die Tiefe geht (Halpern 2014; Paul und Elder 2014; Stanovich 2016). Dies betrifft beispielsweise die Organisation, Evaluation und Nutzung von Wissen (Greene und Yu 2016), den Umgang mit Normen, Werten und Emotionen (Paul und Elder 2014) oder die Erarbeitung und den Einsatz notwendiger Fähigkeiten (Bailin et al. 1999b; Halpern 2014; Paul und Elder 2014).

\section{Wissen}

Beim KD werden kontextspezifisches Wissen sowie ein konzeptuelles Verständnis der bedachten Thematik benötigt, damit beispielsweise intellektuelle Standards wirken 
und Fähigkeiten eingesetzt werden können (Bailin et al. 1999a, b; Bailin 2002; Ennis 1990; Halpern 2014; Lipman 1988; McPeck 1981; Viennot und Décamp 2016). Es gibt empirische Hinweise, dass sich ein konzeptuelles Verständnis und eine kritische Haltung gegenseitig begünstigen (Viennot und Décamp 2016). KD bedeutet unter anderem darüber zu reflektieren, was man weiß, woher dieses Wissen kommt und inwiefern dieses gerechtfertigt und bedeutsam ist (Greene und Yu 2016; Kuhn 1999; Schraw et al. 2006; vgl. Intellektuelle Standards; vgl. Selbstregulation). Dabei muss ein kritischer Denker bzw. eine kritische Denkerin ,dauerhafte Institutionen und in diese eingelagerte Wissensformen und -bestände“ respektieren lernen, „,deren Kontinuität auch zum Zwecke der Kritik es zu sichern gilt" (Winch 2003, S. 30). Dazu gehört beispielsweise der Energieerhaltungssatz, eine aus Atomen aufgebaute Materie oder das Verständnis, dass die naturwissenschaftliche Erkenntnisgewinnung bestimmten Werten und Prinzipien folgt. Kritische Denkerinnen und Denker müssen verstehen, dass naturwissenschaftliches Wissen nur begründet und nicht willkürlich veränderbar ist. In diesem Bereich ergeben sich Parallelen zu Nature of Science (s. Abschn. 5).

\section{Fähigkeiten}

$\mathrm{KD}$ ist nur im Handeln erkennbar, weshalb es notwendig ist zu eruieren, was ein kritischer Denker oder eine kritische Denkerin leisten soll (Bailin et al. 1999b). Beim KD werden kognitive Fähigkeiten benötigt (Bailin et al. 1999b; Ennis 1996; Dwyer et al. 2014; Facione 1990; Halpern 2014). Dennoch ist KD nicht als Kompetenz oder ein Bündel von Fähigkeiten aufzufassen, das generell auf andere Kontexte, als jene, in denen es erworben wurde, übertragbar ist (Abrami et al. 2015; Bailin et al. 1999a, b; Lipman 1988; Winch 2003).

Kritische Denkerinnen und Denker sind fähig zur Synthese (lernen, recherchieren, zusammenfassen, messen, beobachten), Determination (definieren, systematisieren, kategorisieren, ordnen, planen), Reflexion (auseinandersetzen, überlegen), Interpretation (verstehen, ableiten, übertragen, vernetzen), Evaluation (bewerten, hinterfragen, analysieren) und Diskussion (argumentieren, debattieren, kommentieren) (Facione 1990; Paul und Elder 2014; Halpern 2014). Die hier vorgeschlagenen Begriffe orientieren sich an den Naturwissenschaften und können im jeweiligen Lernumfeld, beispielsweise bei der Beschäftigung mit Socio-Scientific Issues, durch andere Begriffe ersetzt oder ergänzt werden. Die Fähigkeiten überschneiden sich mit der Bloom'schen Taxonomie von Lernzielen (Bloom et al. 1974) und den Handlungsaspekten von Kompetenzmodellen, wie die Aneignung, Kommunikation und Organisation von Fachwissen, die Erkenntnisgewinnung sowie das Ziehen von Konsequenzen, das Begründen von Standpunkten und reflektiertes Handeln (BGBl 2018; EDK 2011; KMK 2005; Vieira et al. 2011).

\section{Haltung und Motivation}

Kritische Denker und Denkerinnen brauchen in der Auseinandersetzung mit einem Objekt oder Subjekt ein hohes $\mathrm{Maß}$ an Motivation, genauso wie gewisse Dispositionen, Einstellungen oder Haltungen (Dwyer et al. 2014; Ennis 1996; Facione 1990; Foucault 1992; Kuhn 1999; Paul und Elder 2014; Siegel 1988). Beim KD soll unter anderem ausdauernd, differenziert, fundiert, rational, wahrheits- und zielorientiert sowie vernunftbasiert nach Wissen, Expertise, Erkenntnis oder Problemlösungen gestrebt werden (Facione 1990; Halpern 2014; Paul und Elder 2014; Siegel 1988). KD braucht die Bereitschaft, das eigene Denken $\mathrm{zu}$ optimieren, zu kontrollieren und zu reflektieren (vgl. Selbstregulation). Dazu gehören neben dem Streben nach Wissen, Expertise, Erkenntnis, Wahrheit und Problemlösung unter anderem auch Rationalität, Vernunft, Offenheit, Mut, Verantwortungsbewusstsein, Selbstbewusstsein sowie Selbstwirksamkeit, Bescheidenheit, Kreativität, Neugierde, Frustrationstoleranz und Ausdauer (Bailin 1999b; Bandura, 1997; Ennis 1996; Halpern 2014; Kuhn 1999; Paul und Elder 2014; Siegel 1988; Stanovich 2016). Zudem müssen kritische Denkerinnen und Denker analysieren, welche Motivation und Haltungen hinter dem Objekt oder Subjekt, mit dem sie involviert sind, stehen können. Genauso müssen sie ihre eigene Haltung, Einstellung und Motivation reflektieren (vgl. Selbstregulation). Kritische Denker und Denkerinnen sind sich der Bedeutung von Haltungen und Motivation in der Wissenschaft und in der Gesellschaft bewusst und verstehen, welche Aspekte diese beeinflussen (z.B. Deci und Ryan 1985).

\section{Normen, Werte und Emotionen}

Das Konstrukt KD, und damit auch das hier vorgeschlagene Synergiemodell des kritischen Denkens, ist selbst normativ limitiert (Bailin et al. 1999b). KD umfasst nicht nur die realen mentalen Prozesse des Denkens, sondern auch dessen normative und erwünschte Qualitäten. Neben den intellektuellen Standards gibt es weitere Kriterien, die das Involviertsein mit dem Objekt oder Subjekt kontrollieren sollen. Normen, Definitionen, Gesetze und Konventionen (Lipman 1988) regeln das gesellschaftliche Zusammenleben und die naturwissenschaftliche Wissensgenese (s. Abschn. 5). Sie bilden Grenzen, deren Überschreitung gesellschaftlich oder strafrechtlich geahndet werden kann. Der Verkauf von menschlichen Organen ist verboten. Menschen, die aufgrund ihrer Hautfarbe diskriminiert werden, können dagegen rechtliche Mittel ergreifen. Die fehlende Replizierbarkeit von naturwissenschaftlichen Methoden gilt als 
Qualitätsmangel und führt allgemein zur Ablehnung sich daraus ableitender Erkenntnisse. Hier offenbart sich ein Dilemma. Einerseits soll beim KD sichergestellt werden, dass Normen und Werte beachtet werden. Andererseits können sich die Normen und Werte nicht zuletzt durch KD ändern. Deshalb bedarf es eines andauernden kultur- und gesellschaftssensiblen sowie kontextspezifischen Diskurses über mögliche Veränderungen. Beispielsweise gibt es nicht nur aktuell in unterschiedlichen Ländern verschiedene Regelungen zum Schwangerschaftsabbruch, sondern die Gesetzeslage hat sich in vielen Ländern im Rahmen von weitreichenden gesellschaftlichen Veränderungen gewandelt.

Ferner sind sich kritische Denker und Denkerinnen der Bedeutung von Normen, Werten und Emotionen in der Wissenschaft und in der Gesellschaft bewusst und verstehen, inwiefern sich diese gegenseitig beeinflussen. Genauso verstehen sie, inwiefern diese die eigene Position und fremde Positionen beeinflussen (Paul und Elder 2014). Beispielsweise kann der Verzehr bestimmter Tiere aus emotionalen Gründen abgelehnt werden oder die chemische Industrie negativ wahrgenommen werden, da bestimmte Phänomene, wie Umweltgifte, mit ihr in Verbindung gebracht werden. Dafür braucht es die Fähigkeit und die Bereitschaft zur Empathie (Facione 1990; vgl. Selbstregulation) und Verantwortungsbewusstsein (Lipman 1988). Diese Überlegungen werden auch im Kontext des Konzepts Socio-Scientific Issues diskutiert (s. Abschn. 5).

\section{Selbstregulation}

Die Selbstregulation hat eine zentrale Rolle im Synergiemodell des kritischen Denkens. In wissenschaftlicher Literatur wird die Selbstregulation explizit als ein wichtiger Aspekt von KD genannt (z. B. Dwyer et al. 2014; Facione 1990; Pithers und Soden, 2000). In der Psychologie bezieht sich der Begriff Selbstregulation auf jene Fähigkeit, das eigene Verhalten zielgerichtet $\mathrm{zu}$ beobachten, zu bewerten, zu verändern und damit zu kontrollieren (vgl. Bandura 1991; Kanfer et al. 2012; Schraw et al. 2006). Auch beim KD muss der Ist-Zustand kriteriengeleitet analysiert und bewertet werden, um dann, wenn nötig, eine Korrektur vorzunehmen (Dwyer et al. 2014; Greene und Yu 2016; Facione 1990; Kuhn 1999; Lipman 1988). Hier beschreibt die Selbstregulation also auch den Prozess, sich erneut mit erreichten Positionen auseinanderzusetzen, um sie gegebenenfalls zu revidieren oder zu modifizieren. Dazu müssen sich kritische Denker und Denkerinnen von ihrem System an Emotionen, Haltungen Überzeugungen sowie Wissensformen und -beständen distanzieren, um neue Perspektiven einnehmen zu können. Die Selbstregulation betrifft somit nicht nur die Positionierung, sondern auch das kriterienge- leitete Involviertsein mit dem Objekt oder Subjekt an sich und damit unter anderem

- das genutzte Wissen (z. B. Weiß ich genug? Hat sich der wissenschaftliche Konsens geändert?),

- die angewandten Fähigkeiten (z.B. Wurde die Statistik korrekt interpretiert? Ist die Recherche fair? Ist die Argumentation rational und logisch?),

- die eigene Haltung (z.B. Gehe ich gerade den einfachen Weg? Habe ich den Mut, meine Beweggründe und die von anderen zu hinterfragen?),

- die emotionale Verbindung mit einem Aspekt (z.B. Ekel oder Angst vor bestimmten Tieren) oder

- die Normen und Werte eines Systems (z.B. Wie begründen sich die verschiedenen Rechtslagen zur Stammzellenforschung?).

Kritische Denkerinnen und Denker können und möchten erkennen, wo sie in ihrem Wissen sowie in ihren kognitiven Fähigkeiten, Haltungen, Emotionen und Werten stehen (Dwyer et al. 2014; Schraw et al. 2006). Sie müssen dazu fähig und bereit sein, andere Perspektiven einzunehmen und sich in andere hineinzuversetzen. Wenn Neues gelernt wird oder sich die Faktenlage ändert, muss dies akzeptiert und eigene Unzulänglichkeiten müssen reflektiert werden. Diese Fähigkeit und Bereitschaft braucht es auch in der Auseinandersetzung mit Aussagen, Werten und Handlungen von anderen, sei es von Individuen, Gruppen oder Institutionen. Der Begriff Selbstregulation fasst im Synergiemodell des kritischen Denkens also sowohl die Fähigkeit als auch die Bereitschaft zur Empathie, zur Einsicht und zur Adaption zusammen (Facione 1990; Halpern 2014; Paul und Elder 2014; Sadler und Zeidler 2005).

\section{Kritisches Denken als (curriculares) Bildungsziel im naturwissenschaftlichen Unterricht}

Seit Beginn des 20. Jahrhunderts gibt es curriculare Überlegungen zu KD und dessen Bildungsgehalt, vor allem im anglo-amerikanischen Raum (Abrami et al. 2015; Kennedy et al. 1990). In diesem ist KD fester Bestandteil naturwissenschaftlicher Curricula (Bailin et al. 1999b; Gunn et al. 2008). Auch das Europäische Parlament und der Europäische Rat stufen KD als einen wichtigen Faktor für den nachhaltigen Erwerb der Schlüsselkompetenzen für lebensbegleitendes Lernen ein (Europäische Union 2006, S. 14). Allerdings wird KD damit gleichgesetzt kritisch zu sein und etwas kritisch zu tun (Europäische Union 2006, S. 5, 7). Dasselbe Vorgehen findet sich im österreichischen Lehrplan (BGBl 2018). In diesem gehört KD zu den allgemeinen didaktischen Grundsätzen: 
Die Schülerinnen und Schüler sind ihrem Alter entsprechend $\mathrm{zu}$ kritischem und eigenverantwortlichem Denken zu führen. Es sind Impulse zu setzen, die die Entwicklung eigener Wert- und Normvorstellungen bei den Schülerinnen und Schülern anregen und fördern. (BGBl 2018, S. 15)

Ferner sind Schülerinnen und Schüler ,,zur aktiven Aneignung, aber auch zu einer kritisch-prüfenden Auseinandersetzung mit verfügbarem Wissen zu befähigen und zu ermutigen“, wobei sie ,sich in altersadäquater Form mit Problemstellungen auseinander setzen, Gegebenheiten kritisch hinterfragen, Probleme erkennen und definieren, Lösungswege eigenständig suchen und ihr eigenes Handeln kritisch betrachten" sollen (BGB1 2018, S. 10).

Wie im österreichischen Lehrplan wird der Begriff kritisch auch in den deutschsprachigen Biologie-Lehrplänen der gymnasialen Oberstufen in Deutschland und der Schweiz in unterschiedlicher Häufigkeit und Ausprägung aufgegriffen (Rafolt et al. 2018). Kritisch sein sollen Schülerinnen und Schüler vor allem gegenüber Wissen, Theorien, Modellen, Daten, Quellen, Medien, Vermutungen, Wertvorstellungen und Ideologien bzw. etablierten Systemen im Allgemeinen. Genauer betrachtet sollen sie diese Objekte kritisch hinterfragen oder kritisch beurteilen, aber auch kritisch reflektieren und sich ganz allgemein mit diesen kritisch auseinandersetzen (ebd.). Beispielsweise sollen Schülerinnen und Schüler „Daten, Fakten und Ergebnisse aus verschiedenen Quellen sowie Schlussfolgerungen kritisch hinterfragen und Gründe für deren Annahme oder Verwerfung angeben“ (BGBl 2018, S. 179) oder in der Lage sein ,naturwissenschaftliche Berichte und Meldungen der Medien $\mathrm{zu}$ verstehen und sich kritisch mit ihnen auseinanderzusetzen“" (BGB1 2018, S. 441). Die meisten deutschsprachigen Biologie-Lehrpläne sprechen KD ansatzweise, fragmentiert und interdisziplinär an (Rafolt et al. 2018). Einerseits enthalten die Kompetenzmodelle, die den Lehrplänen zugrunde liegen, einzelne Aspekte von KD (vgl. BGBl 2018; EDK 2011; KMK 2005). Im Vergleich mit dem Synergiemodell des kritischen Denkens (Abb. 1) oder dem „Framework for promoting student's critical thinking in science classrooms" von Vieira und Kolleginnen (2011, S. 50) werden diese Überschneidungen deutlich. Andererseits ist dies auf die Struktur einiger Lehrpläne, besonders der österreichischen, zurückzuführen, die interdisziplinäre pädagogische Ziele von fachspezifischen Bildungszielen trennen. Die Ansätze von KD werden womöglich als allgemeine pädagogisch-didaktische Konzepte betrachtet und deshalb auch nicht systematisch im fachspezifischen Abschnitt vorgestellt.

Wenn es der gesellschaftliche Auftrag von Schule sein soll, KD erfahrbar zu machen, brauchen Lehrkräfte Hinweise aus der Unterrichtsforschung, was KD ist und wie sie zentrale Aspekte erkennen, in Beziehung setzen und entsprechend vermitteln können. In modernen Gesellschaften offenbart sich dabei ein, in der Pädagogik allgegenwärtiges, Dilemma (vgl. Brezinka 1978), das Winch (2003, S. 28) wie folgt beschreibt:

Zum Auftrag ihrer Bildungssysteme gehört es, durch staatsbürgerliche und individuelle Erziehung die Entstehung ,kritischen Denkens“ zu fördern. Während viele diese Aufgabenstellung für uneingeschränkt wünschenswert halten, haben einige bemerkt, dass sie auch zu nicht beabsichtigten Nebenwirkungen führen kann. Eine gegenüber Autoritäten aller Art skeptische Geisteshaltung kann grenzenlos sein. Ein kritischer Arbeiter kann die Kontrolle über das gesamte Unternehmen anstreben, ein kritischer Bürger für die Umwälzung des politischen Systems kämpfen, ein kritisches Individuum der traditionellen Moral den Kampf ansagen und für das Ende aller kollektiven Werte eintreten. (Winch 2003, S. 28)

Ferner wirft Winch (2003) die Frage auf, ob die Folgen des oben beschriebenen Rebellierens tatsächlich „nicht beabsichtigt[e] Nebenwirkungen“ (28) oder vielmehr das Ziel von Bildung sind. Diese Überlegung ist in Hinblick auf die gesellschaftliche Dimension naturwissenschaftlichen Unterrichts elementar. Naturwissenschaftlicher Unterricht soll neben der Vermittlung von Inhalten auch Schwerpunkte in der Auseinandersetzung mit gesellschaftlich relevanten naturwissenschaftlichen Problemstellungen (BGB1 2018) sowie in der Vermittlung eines Verständnisses von Nature of Science setzen (Heering und Kremer 2018).

Unsere Wissensgesellschaft ist gekennzeichnet durch Innovationsdynamiken sowie grenzüberschreitende Informations- und Kommunikationsströme (Heidenreich 2003; Winch 2003). Das Internet erlaubt jederzeit Zugang zu einer unüberschaubaren Menge an Information, dessen Verlässlichkeit und Vertrauenswürdigkeit von der Leserschaft bewertet werden muss. In dieser stark vernetzten Welt des schnellen Wandels werden deshalb der naturwissenschaftlichen Bildung und, daraus abgeleitet, der Fähigkeit, sich mit der Gültigkeit und Verlässlichkeit von Wissen eigenverantwortlich auseinandersetzen zu können, sowie dem Streben nach Wahrheit eine zentrale Bedeutung zuerkannt (Heidenreich 2003; OECD 2018; Winch 2003). Dabei gilt es zu verstehen, dass naturwissenschaftliches Wissen nicht willkürlich ist, sondern auf empirisch gewonnenen Erkenntnissen beruht, welche geprüft und reproduziert werden können. KD fordert die systematische Überprüfung der Belastbarkeit von Wissensbeständen ein. Wissen kann sich im Lauf der Zeit verändern, aber nur, wenn es diesen Belastungstests nicht standhält. In so einem Fall müssen neue Perspektiven in Betracht gezogen und die Qualität des neuen wie des vorherigen Erkenntnisprozesses 
systematisch bewertet werden. „Ein verantwortungsbewusster Wissenschaftler[*] ist in der Lage, einen Schritt zurück zu tun, über die eigenen Handlungen kritisch zu reflektieren und dies auch verständlich darzulegen" (Sieroka et al. 2018). Lernende sollen erfahren, dass Charakteristika naturwissenschaftlicher Erkenntnisprozesse für alltägliche Entscheidungsprozesse hilfreich sein können.

\section{Kritisches Denken im Kontext von Naturwissenschaft und Gesellschaft}

In naturwissenschaftsdidaktischer Literatur wird diskutiert, dass KD einen Rahmen für die Auseinandersetzung mit sogenannten Socio-Scientific Issues und dem Konzept Nature of Science bildet (z. B. Yacoubian 2015). Gleichzeitig gibt es Hinweise darauf, dass die Auseinandersetzung mit Socio-Scientific Issues zum KD anregen (z. B. Klosterman und Sadler 2010; Zeidler und Nichols 2009) und ein Verständnis von Nature of Science begünstigen (vgl. Zeidler 2014).

\section{Socio-Scientific Issues (SSI)}

Naturwissenschaftlicher Unterricht soll die Auseinandersetzung mit gesellschaftlich relevanten Problemen, die naturwissenschaftliche Aspekte beinhalten, fördern (BGB1 2018), beispielsweise indem Schülerinnen und Schüler die „Sachverhalte und Probleme unter Einbeziehung kontroverser Gesichtspunkte reflektiert erörtern und begründet bewerten“ oder die „Bedeutung, Chancen und Risiken der Anwendungen von naturwissenschaftlichen Erkenntnissen für das Individuum und für die Gesellschaft erkennen, um verantwortungsbewusst zu handeln“ (BGB1 2018, S. 173).

Naturwissenschaftliche Themen mit hoher Relevanz für die Gesellschaft werden in der naturwissenschaftsdidaktischen Literatur häufig als Socio-Scientific Issues (SSI) bezeichnet. SSI sind ergebnisoffen, facettenreich, lebensnah sowie herausfordernd und können ein soziales oder moralisches Dilemma provozieren (für einen Überblick siehe: Zeidler 2014). Das Konzept SSI umfasst nicht nur die Beziehungen zwischen Naturwissenschaften, Gesellschaft und Technologie, sondern auch deren ethischen und moralischen Dimensionen (Zeidler et al. 2005, 2009). Es bietet einen theoretisch fundierten Rahmen für Lehrende und die fachdidaktische Forschung, der auch entwicklungspsychologische und soziologische Forschung beinhaltet und somit die emotionale Reife, epistemologische Überzeugungen und die soziale Identitäts- und Charakterentwicklung von Lernenden berücksichtigt (Zeidler et al. 2002, 2005).

Das Aufgreifen von SSI stellt Lehrkräfte vor ähnliche Herausforderungen, wie sie auch im Kontext von KD diskutiert werden. Eine fundierte Auseinandersetzung mit SSI erfordert unter anderem naturwissenschaftliches Wissen, ethi- sches Bewerten sowie die Reflexion eigener Werte und Emotionen (Zeidler 2014). Bei vielen SSI gibt es keine eindeutigen Antworten und Handlungsempfehlungen, was wohl viele Schülerinnen und Schüler, aber auch Lehrkräfte vom traditionellen naturwissenschaftlichen Unterricht nicht gewohnt sind (Fensham 2012). Zeidler und Nichols (2009) empfehlen, dass sich Lehrkräfte beim Aufgreifen von SSI im naturwissenschaftlichen Unterricht auf aktuelle Recherchen und Informationen verlassen, um Debatten im Unterricht durch themenspezifische und epistemologische Fragestellungen, moralisches Argumentieren sowie durch Übungen im Rollentausch besser lenken zu können. Eine verantwortungsbewusste Auseinandersetzung setzt ein umfassendes Wissen voraus, das Lehrenden erst ermöglicht die Qualität der Argumente und Erklärungen, die Lernende in den Diskurs einbringen, zu bewerten und so Lernfortschritte zu unterstützen. Im Kontext von SSI gilt es daher zu berücksichtigen, dass KD und Wissen sowie ein Verständnis von Wissensgenesen zwar Voraussetzungen für ein selbstbestimmtes Leben in einer rationalen und demokratischen Gesellschaft sind (Bailin et al. 1999a; Facione 1990; Winch 2003; Vieira et al. 2011), aber auch Quellen für Verunsicherung. Wissenszuwachs kann zur Erkenntnis führen, dass der bisherige Wissenstand begrenzt oder falsch war (Heidenreich 2003; Winch 2003). Lehrkräfte müssen hier selbst entsprechend der Kriterien von KD agieren und Rückmeldungen geben können.

\section{Nature of Science}

Bei der Betrachtung des Synergiemodells des kritischen Denkens (vgl. Abb. 1) stellen sich zwangsläufig Fragen, wie:

Welches Wissen wird beim KD im Kontext des naturwissenschaftlichen Unterrichts benötigt und wie sehr muss dieses sowohl in die Breite als auch in die Tiefe gehen?

Wie viel Mündigkeit wird vom Individuum beim KD erwartet und diesem tatsächlich zugestanden?

Weil die naturwissenschaftlichen Fächer und Fachdidaktiken seit Jahrzehnten, vorwiegend im anglo-amerikanischen Sprachraum, neben der Vermittlung von Inhalten einen weiteren Schwerpunkt im Bereich der Vermittlung eines Verständnisses von Nature of Science legen (für einen Überblick siehe: Heering und Kremer 2018), sehen wir im disziplinären Kontext der Naturwissenschaftsdidaktiken ein fruchtbares Feld für die Beantwortung dieser drängenden Fragen.

Nature of Science bezieht sich auf die Charakteristika naturwissenschaftlichen Wissens und naturwissenschaftlicher Erkenntnisgewinnung, sowie auf die Wertebasis, auf der naturwissenschaftliche Entscheidungen gründen (McComas und Olson 1998). Während frühe Konzeptualisierungen von Nature of Science (Lederman et al. 2002) die 
rationalen Kriterien der naturwissenschaftlichen Wissensgenese etwas mehr betonen, werden die Eigenschaften der naturwissenschaftlichen Wissensgenese in neueren Konzeptualisierungen (Allchin 2012; Erduran und Dagher 2014) verstärkt im Sinne der Gratwanderung zwischen rationalistischen und subjektivistischen Positionen betont (vgl. auch: Neumann und Kremer 2013).

Erduran und Dagher (2014) betrachten die Naturwissenschaften als kognitiv-epistemisches und als sozial-institutionelles System. Im kognitiv-epistemischen Bereich bedeutet es, dass naturwissenschaftliche Erkenntnisgewinnung an den Kriterien der kritischen Rationalität (vgl. Popper 1984) orientiert ist. Naturwissenschaftlerinnen und Naturwissenschaftler durchlaufen einen Prozess der Erkenntnisgewinnung, der bestimmte Elemente enthält (z.B. Fragestellung, Hypothesenbildung, Dateninterpretation). Dabei orientieren sie sich an den epistemischen Gütekriterien wissenschaftlichen Arbeitens (Reproduzierbarkeit, Konsistenz, Vorhersagbarkeit, etc.) und wenden vielfältige systematische Ansätze bzw. Regeln an (z.B. das induktive und deduktive Schlussfolgern, das Berücksichtigen von Kontrollansätzen beim Testen von kausalen Hypothesen). Andererseits ist die institutionelle Ebene der naturwissenschaftlichen Wissensproduktion ein von Menschen gemachtes Unterfangen. Wer die Eigenschaften naturwissenschaftlicher Erkenntnisgewinnung auch im sozialen und historischen Kontext umfassend begreifen möchte, muss demnach dessen spezifische Elemente (professionelle Kommunikation, Peer Review, Forschungsfinanzierung, politische Strömungen) verstehen (vgl. auch: Heering und Kremer 2018).

KD bedeutet unter anderem das eigene Wissen und dessen Rechtfertigung zu reflektieren (Kuhn 1999). Welchen Regeln die Auseinandersetzung mit Wissen folgt und welchen Qualitätskriterien KD unterworfen ist, ist immer an den sozialen Kontext sowie an die Normen und Werte der Scientific Community gebunden. Lernende sollen verstehen, dass Kritik und Zweifel nicht nur Randmerkmale der Wissenschaft sind, sondern den Kern ihrer Praxis bilden und der Aufbau zuverlässigen Wissens ohne Argumentation und Bewertung unmöglich ist (eigene Übersetzung: Osborne 2014, S. 53: Ford 2008).

Eine explizite Betrachtung von Nature of Science aus kognitiv-epistemischer und aus sozialer Perspektive im naturwissenschaftlichen Unterricht erscheint vor dem Hintergrund der aktuellen gesellschaftlichen Diskurse besonders hilfreich, um bei Lernenden grundlegende Wissensbestände und Einstellungen über die Naturwissenschaften herauszubilden, die für die Entwicklung und Förderung von KD bedeutsam sind.

\section{Ableitung von Desiderata und Diskussion}

Aus der Schilderung des theoretischen Anspruchs heraus werden im Folgenden Desiderata für eine zeitgemäße Implementierung von KD in naturwissenschaftliche Curricula, im naturwissenschaftlichen Unterricht und in die Lehrkräftebildung sowie in die naturwissenschaftsdidaktische Forschung abgeleitet. Es soll eine Diskussion darüber angestoßen werden, ob und wie naturwissenschaftsdidaktische Forschung Akzente setzen kann, um kontextspezifisch besser verstehen zu lernen, welche Aspekte KD beeinflussen und wie sich ein differenzierteres Verständnis in der unterrichtlichen Praxis niederschlagen kann.

\section{Erstes Desiderat: Fachbezogene Konzeptualisierung}

Der Delphi-Report (Facione 1990) beschreibt KD sehr umfassend, doch nach fast 30 Jahren ist es an der Zeit eine Erklärung zu entwickeln, die

- fachspezifisch ist; Die Unterrichtsfächer Geschichte und Physik sprechen KD vermutlich unterschiedlich an, weil es verschiedene Fachkulturen im Sinne der Auseinandersetzung mit Wissen gibt.

- kultur- und gesellschaftssensibel ist; Da KD normativ bestimmt ist, müssen gesellschaftliche Normen und Werte und deren kulturelle Verankerung in die Reflexion über KD miteinbezogen werden.

- verständlich, nachvollziehbar und anwendbar ist; So kann KD in den naturwissenschaftlichen Unterricht und die Lehrkräftebildung integriert werden.

Das vorgestellte Synergiemodell des kritischen Denkens (Abb. 1) bietet diese Erklärung. Es sollte künftig auch für eine empirische Prüfung herangezogen werden, eine Operationalisierung der Konstrukte fehlt jedoch bisher. Auch wenn es für einzelne Aspekte von KD bereits erprobte Messinstrumente gibt, können nicht alle Charakteristika und vor allem nicht deren Synergieeffekte direkt beobachtet werden. Jene Tests, die darauf abzielen verschiedene Niveaus einzelner Aspekte von KD, wie logisches Schlussfolgern oder Argumentieren, zu ermitteln (für einen Überblick siehe: Tiruneh et al. 2017), vernachlässigen die systemische Dimension von KD (vgl. Abb. 1). Die Ermittlung einzelner Fähigkeiten zeichnet kein Gesamtbild über die Ausprägung von KD.

Fachdidaktische Forschung kann für eine Weiterentwicklung des Forschungsgebietes wertvolle Beiträge leisten. Es gibt kaum Studien, die sich mit der komplexen Umsetzung des Bildungsziels KD in realen Lehr-Lernumgebungen befassen. Kontextunabhängig erklärt das Synergiemodell des kritischen Denkens aktuell nicht, welche Aspekte wichtiger oder effektstärker sind. Es braucht in weiterer Folge eine fachdidaktische Ausschärfung, die eine fachspezifische 
Auseinandersetzung mit diesem Bildungsziel in Forschung und Praxis ermöglicht.

Die naturwissenschaftsdidaktische Forschung beschäftigt sich im Bereich SSI intensiv mit Aspekten, die auch im Kontext von KD diskutiert werden müssen. Dazu gehört die Erforschung der Einflüsse moralischer Überzeugungen, emotionaler Reife, persönlicher Erfahrungen, inhaltlichen Wissens und Fähigkeiten des naturwissenschaftlichen Denkens (z. B. Fowler et al. 2009; Sadler und Zeidler 2005; Zeidler et al. 2009, 2002). Neben den im Artikel diskutierten Konzepten SSI und Nature of Science, beschäftigen sich Arbeiten im Bereich des Argumentierens (z. B. Jiménez-Aleixandre und Puig 2012), des Bewertens (z. B. Bögeholz et al. 2004; Dittmer et al. 2016), oder des Verstehens (z.B. Combe und Gebhard 2012), die Facetten von idealtypischen KD sind, genauso wie die Psychologie (z.B. Kuhn 1999; Schraw et al. 2006) mit Fragestellungen, die sich auch bei der Auseinandersetzung mit dem Konstrukt KD stellen. Wie beeinflusst das Zusammenspiel der Einzelfaktoren, also Synergieeffekte, die Qualität von KD? Wie sind Lernumgebungen zu gestalten, damit Lernende genuine Erfahrung mit KD machen können? Hier sollten künftig Verknüpfungen hergestellt werden. Auf diese Weise erhalten aktuell noch in unterschiedlichen Strömungen verortete Forschungsansätze ein übergeordnetes Dach.

\section{Zweites Desiderat: Möglichkeiten und Grenzen}

Im Alltag begegnen Schülerinnen und Schüler gesellschaftlichen Herausforderungen, die eine Auseinandersetzung mit aktuellen naturwissenschaftlichen Wissensbeständen erfordern. Das Konzept SSI bietet einen Rahmen, um diese Themen im naturwissenschaftlichen Unterricht aufzugreifen. Dafür brauchen alle Beteiligten eine adäquate naturwissenschaftliche Bildung (Scientific Literacy), ein Verständnis der Nature of Science und KD (Bybee 1997; Gunn et al. 2008; Kolst $\varnothing$ et al. 2006; Osborne 2014; Yacoubian 2015; Zeidler 2014). SSI liegen selten eindeutige Antworten und Handlungsempfehlungen zugrunde, was Schülerinnen und Schüler, aber auch Lehrkräfte verunsichern und in der unterrichtlichen Praxis vor Herausforderungen stellen kann. Wir sehen im Synergiemodell des kritischen Denkens (s. Abb. 1) für die Naturwissenschaftsdidaktik eine Möglichkeit diesen zu begegnen, beispielsweise indem Unsicherheiten und Herausforderungen kontextspezifisch analysiert werden und daraus Vorschläge für förderliche Lern-Lehrumgebungen erbracht werden.

KD erzeugt ein Dilemma, da einerseits Normen und Werte beachtet werden sollen und andererseits diese nicht zuletzt durch KD hinterfragt werden oder sich sogar ändern können. Während der Auseinandersetzung mit weitgehend ergebnisoffenen SSI sollen Schülerinnen und Schüler sowie deren Lehrkräfte ihre Denkinstrumente benützen und dabei sowohl die Aspekte von KD als auch deren Synergieeffekte beachten (vgl. Abb. 1). Eine autoritär-dogmatische Haltung ist jedenfalls nicht sinnvoll, weil diese die Position des Gegenübers nicht zulässt. Das bedeutet nicht, dass beispielsweise ein Eintreten „für das Ende aller kollektiven Werte" (Winch 2003, S. 28) oder das Verbreiten von Verschwörungstheorien unter dem Deckmantel von KD akzeptiert werden muss. Das Synergiemodell des kritischen Denkens zeigt auf, wie herausfordernd KD ist und hilft zu verstehen, welche Aspekte bei der Auseinandersetzung mit einer Thematik womöglich noch gar nicht bedacht wurden (vgl. Rafolt und Kapelari 2018). Unter der von Winch (2003, S. 28) erwähnten „nicht beabsichtigten Nebenwirkungen“" von KD verstehen wir die Gefahr, dass bei dem Involviertsein mit einem Objekt oder Subjekt (vgl. Abb. 1) auf halbem Weg stehen geblieben wird und die erreichte Position dennoch mit dem Prädikat KD ausgezeichnet wird. Dabei fehlt entweder ein differenziertes Verständnis von KD oder die dafür notwendige Haltung und Motivation sowie die Möglichkeiten und Grenzen von KD im Kontext naturwissenschaftlichen Wissens, genauso wie dessen Bedeutung für gesellschaftliche Herausforderungen, wurden nicht reflektiert. Wir plädieren für eine sensible Implementierung von KD in den naturwissenschaftlichen Unterricht und die Lehrkräftebildung. Naturwissenschaftlicher Unterricht, der SSI ins Zentrum rückt und KD erfahrbar machen möchte, erschöpft sich nicht im Kundtun oberflächlicher Haltungen und Meinungen. Es braucht Lehrkräfte, die ein undifferenziertes Verständnis von KD erkennen und entsprechend reagieren.

\section{Drittes Desiderat: Sensible Verankerung in den Lehrplänen und der Lehrkräftebildung}

Die Clearing House Unterricht Research Group der Technischen Universität München sieht in der Metaanalyse von Abrami und Kollegen (2015) ,ein solides Argument dafür, dass es in erster Linie wichtig ist, dass Lehrkräfte das Fördern von kritischem Denken überhaupt zu einem erklärten Unterrichtsziel machen" (Hetmanek et al. 2018, S. 5). $\mathrm{Ob}$ und in welcher Qualität KD in den Unterricht einfließt und somit Schülerinnen und Schülern vermittelt werden kann, hängt jedoch auch vom Verständnis der Lehrkräfte $a b$. Diese brauchen eine klare Vorstellung von KD und jenen Handlungen, die auf KD hinweisen (Ab Kadir 2017; Bailin et al. 1999b; Lipman 1988). Dabei darf die Implementierung von KD in den naturwissenschaftlichen Unterricht nicht mit dem Abarbeiten einer Liste von „Critical Thinking Skills“ (Facione 1990, S. 3) gleichgesetzt werden (Bailin et al. 1999a, b; Winch 2003). So könnten Versuche, Schülerinnen und Schüler oder Lehrkräfte zum KD anzuregen, zu oberflächlichen und kurzweiligen Scheinhandlungen werden, wenn die Beteiligten nicht genau wissen, was 
damit eigentlich erreicht werden soll (Ab Kadir 2017; Bailin 2002). Deshalb braucht es eine sensible und explizite Verankerung von KD in der Lehrkräftebildung und in Lehrplänen sowie nationalen Bildungsstandards als normative Ausgangspunkte für naturwissenschaftlichen Unterricht. Es genügt nicht KD phrasenhaft oder als reines Schlagwort zu erwähnen, ohne auf den systematischen Bezug zum Fachwissenserwerb oder auf eine explizite Erklärung der Möglichkeiten und Grenzen des Konstrukts zu verweisen, denn wir wissen kaum etwas darüber, was Lehrkräfte unter KD verstehen, welche Chancen und Herausforderungen sie dabei sehen und wie sie KD bei Lernenden beobachten (vgl. Hasslöf und Malmberg 2015; Rafolt und Kapelari 2018).

Wenn Schülerinnen und Schüler gegenüber Wissen, Theorien, Modellen, Daten, Quellen, Medien, Vermutungen, Wertvorstellungen oder Ideologien kritisch sein sollen (Rafolt et al. 2018), brauchen Lehrkräfte ein differenziertes Verständnis von KD. Sie müssen verstehen, dass sich KD von ähnlichen Nominalphrasen, wie kritisch sein oder etwas kritisch tun, unterscheidet. Das ist insbesondere wichtig, wenn Kritik als ,eine allgemein prüfende Einstellung gegenüber selbstverständlichen Wissensbeständen in allen Bereichen menschlicher Erfahrung " verstanden wird (Lembeck 1996, S. 279) und zudem mit (An-)Zweifeln assoziiert wird sowie negativ konnotiert ist (Halpern 2014; Röttgers 1975; Schneiders 1985). Ein differenziertes Verständnis von KD reduziert sich nicht auf Skepsis und Ablehnung (vgl. Abb. 1). Das Synergiemodell des kritischen Denkens bietet die Möglichkeit einem undifferenzierten Verständnis im naturwissenschaftlichen Unterricht entgegen zu wirken, da es aufzeigt, wie umfassend, differenziert und anspruchsvoll KD ist. Es hilft dabei sich über Charakteristika von $\mathrm{KD}$ und deren Bedeutung kontextspezifisch sowie kultur- und gesellschaftssensibel zu verständigen. Lehrkräfte können ihre Haltungen und jene der Lernenden reflektieren und förderliche Bedingungen für die Entwicklung von KD im naturwissenschaftlichen Unterricht in Hinblick auf die vorherrschenden Lehr-Lernbedingungen eruieren. Die Bereitschaft dazu hängt jedoch auch von der individuellen Motivation (Facione 1990; Paul und Elder 2014) und kritischen Haltung (Foucault 1992; Siegel 1988) ab. Die Förderung von KD kann für alle Beteiligten anstrengend und ressourcenintensiv sein. Wenn Schülerinnen und Schüler oder auch Lehrkräfte nicht motiviert sind, sich mit Inhalten ausdauernd, rational, differenziert und fundiert auseinanderzusetzen, sowie ein critical spirit (Ennis 1996; Facione 1990; Siegel 1988) fehlt, ist die Förderung von KD im Unterricht schwierig. Die fachdidaktische Forschung und Angebote in der Lehrkräftebildung müssen daher auch förderliche Lehr-Lernumgebungen fokussieren.

\section{Conclusio}

KD kann nicht auf diskrete mentale Prozesse und Verfahrensschritte reduziert werden, die unabhängig voneinander durch Praxis verbessert und auf andere Bereiche übertragen werden (Bailin et al. 1999a). Ein undifferenziertes Verständnis von KD könnte die Komplexität der benötigten kognitiven Abläufe ignorieren, angestrebte Bildungsziele verfälschen und die fachspezifische Implementierung von KD im naturwissenschaftlichen Unterricht behindern.

Das hier vorgestellte Synergiemodell des kritischen Denkens (vgl. Abb. 1) schafft ein Vermittlungsangebot, indem es wesentlicher Facetten des idealtypischen KD veranschaulicht und einen Referenzrahmen für die Verständigung über Charakteristika von KD in Forschung und Praxis darstellt.

Im Kontext der naturwissenschaftsdidaktischen Forschung hilft das Synergiemodell des kritischen Denkens das Konstrukt themen- und kontextspezifisch auszuschärfen und bereits bestehende Forschungsergebnisse, die Teilaspekte von KD betrachten, miteinander in Beziehung zu setzen. Welchen Einfluss hat bereits vorhandenes Fachwissen auf die Anwendung intellektueller Standards oder/und die Selbstreflexion eines kritisch denkenden Menschen? Steigert ein Lernumfeld, welches das Verknüpfen von Fachinhalten systematisch fördert, die Qualität von KD?

Im Kontext naturwissenschaftlicher Unterrichtspraxis kann dieses Modell dafür genützt werden, Schülerinnen und Schüler, Lehramtsstudierende und Lehrkräfte auf normative und nicht-normative Aspekte in der Auseinandersetzung mit Socio-Scientific Issues vorzubereiten und sichtbarmachen, was es heißt, sich verantwortungsbewusst eine Meinung zu bilden.

Danksagung Wir bedanken uns herzlich bei den anonymen GutachterInnen für die ausführlichen Rückmeldungen und die wertvollen Hinweise.

Funding Open access funding provided by University of Innsbruck and Medical University of Innsbruck.

Open Access Dieser Artikel wird unter der Creative Commons Namensnennung 4.0 International Lizenz (http://creativecommons.org/ licenses/by/4.0/deed.de) veröffentlicht, welche die Nutzung, Vervielfältigung, Bearbeitung, Verbreitung und Wiedergabe in jeglichem Medium und Format erlaubt, sofern Sie den/die ursprünglichen Autor(en) und die Quelle ordnungsgemäß nennen, einen Link zur Creative Commons Lizenz beifügen und angeben, ob Änderungen vorgenommen wurden.

\section{Literatur}

Abrami, P.C., Bernard, R. M., Borokhovski, E., Waddington, D. I., Wade, C. A., \& Persson, T. (2015). Strategies for teaching students to think critically: a meta-analysis. Review of Educational Research, 85(2), 275-314. 
Allchin, D. (2012). Towards clarity on whole science and KNOWS. Science Education, 96(4), 693-700.

Astleitner, H. (1998). Kritisches Denken: Basisqualifikation für Lehrer und Ausbilder. Innsbruck: Wien: Studien-Verlag.

Bailin, S. (2002). Critical thinking and science education. Science Education, 11, 361-375.

Bailin, S., Case, R., Coombs, J. R., \& Daniels, L. B. (1999a). Common misconceptions of critical thinking. Journal of Curriculum Studies, 31(3), 269-283.

Bailin, S., Case, R., Coombs, J. R., \& Daniels, L. B. (1999b). Conceptualizing critical thinking. Journal of Curriculum Studies, 31(3), $285-302$.

Bandura, A. (1991). Social cognitive theory of self-regulation. Organizational Behavior and Human Decision Processes, 50, 248-287.

Bandura, A. (1997). Self-efficacy: the exercise of control. New York: Freeman.

BGBl [Bundesgesetzblatt für die Republik Österreich] (2018). Teil II, Nr. 230/2018. Bundesrecht konsolidiert: Gesamte Rechtsvorschrift für Lehrpläne - allgemeinbildende höhere Schulen. https://www.ris.bka.gv.at/GeltendeFassung.wxe?Abfrage= Bundesnormen\&Gesetzesnummer $=10008568$. Zugegriffen: 17. Mai 2019.

Bittner, R. (2009). Kritik, und wie es besser wäre. In R. Jaeggi \& T. Wesche (Hrsg.), Was ist Kritik? (S. 134-149). Frankfurt a. M.: Suhrkamp.

Bloom, B., Engelhart, M., Furst, E., Hill, W., \& Krathwohl, D. (1974). Taxonomie von Lernzielen im kognitiven Bereich (4. Aufl.). Weinheim: Beltz.

Bögeholz, S., Hößle, C., Langlet, J., Sander, E., \& Schlüter, K. (2004). Bewerten - Urteilen - Entscheiden im biologischen Kontext: Modelle in der Biologiedidaktik. Zeitschrift für Didaktik der Naturwissenschaften, 10, 89-115.

Brezinka, W. (1978). Metatheorie der Erziehung: eine Einführung in die Grundlagen der Erziehungswissenschaft, der Philosophie der Erziehung und der Praktischen Pädagogik. München, Basel: Ernst Reinhardt.

Bybee, R.W. (1997). Achieving scientific literacy: From purposes to practices. Portsmouth: Heinemann.

Combe, A., \& Gebhard, U. (2012). Annäherung an das Verstehen im Unterricht. Zeitschrift für interpretative Schul- und Unterrichtsforschung, 1, 221-230.

Davies, M. (2013). Critical thinking and the disciplines reconsidered. Higher Education Research \& Development, 32(4), 529-544.

Deci, E. L., \& Ryan, R. M. (1985). Intrinsic Motivation and Self-Determination in Human Behavior. New York: Springer.

Dittmer, A., Gebhard, U., Höttecke, D., \& Menthe, J. (2016). Ethisches Bewerten im Naturwissenschaftlichen Unterricht: Theoretische Bezugspunkte. Zeitschrift für Didaktik der Naturwissenschaften, 22, 97-108.

Dwyer, C., Hogan, M., \& Stewart, I. (2014). An integrated critical thinking framework for the 21st century. Thinking Skills and Creativity, 12, 43-52.

EDK [Schweizerische Konferenz der kantonalen Erziehungsdirektoren] (2011). Grundkompetenzen für die Naturwissenschaften. Nationale Bildungsstandards. Frei gegeben von der EDK-Plenarversammlung am 16. Juni 2011

Ennis, R. H. (1990). The extent to which critical thinking is subject specific: further clarification. Educational researcher, 19(4), 13-16.

Ennis, R. H. (1996). Critical thinking dispositions: their nature and assessability. Informal Logic, 18(2\&3), 165-182.

Erduran, S., \& Dagher, Z.R. (2014). Reconceptualizing the nature of science for science education. Scienitifc knowledge, practices and other family categories. New York: Springer.

Europäische Union (2006). Empfehlung des Europäischen Parlaments und des Rates vom 18. Dezember 2006 zu Schlüsselkompetenzen für lebensbegleitendes Lernen. AB1. L 394 vom 30.12.2006. (S. 10-18).
Facione, P. A. (1990). Critical thinking: a statement of expert consensus for purposes of educational assessment and instruction(The Delphy Report). Millbrae: California Academic Press.

Fensham, P.J. (2012). Preparing citizens for a complex world: the grand challenge of teaching Socio-scientific issues in science education. In A. Zeyer \& R. Kyburz-Graber (Hrsg.), Science environment health. Towards a renewed pedagogy for science education (S. 7-29). Berlin: Springer.

Ford, M. J. (2008). Disciplinary authority and accountability in scientific practice and learning. Science Education, 92(3), 404-423.

Foucault, M. (1992). Was ist Kritik? Berlin: Merve.

Fowler, S. R., Zeidler, D.L., \& Sadler, T.D. (2009). Moral sensitivity in the context of socioscientific issues in high school science students. International Journal of Science Teacher Education, 31(2), 279-296.

Greene, J. A., \& Yu, S. B. (2016). Educating critical thinkers: the role of epistemic cognition. Policy Insights from the Behavioral and Brain Sciences, 3, 45-53.

Gunn, T. M., Grigg, L. M., \& Pomahac, G. A. (2008). Critical thinking in science education: can bioethical issues and questioning strategies increase scientific understandings? The Journal of Educational Thought, 42(2), 165-183.

Halpern, D.F. (2014). Thought and knowledge: an introduction to critical thinking. New York: Psychology Press.

Hasslöf, H., \& Malmberg, C. (2015). Critical thinking as room for subjectification in education for sustainable development. Environmental Education Research, 21(2), 239-255.

Heering, P., \& Kremer, K. (2018). Nature of Science. In D. Krüger, I. Parchmann \& H. Schecker (Hrsg.), Theorien in der naturwissenschaftsdidaktischen Forschung (S. 105-119). Heidelberg: Springer Spektrum.

Heidenreich, M. (2003). Die Debatte um die Wissensgesellschaft. In S. Böschen \& I. Schulz-Schaeffer (Hrsg.), Wissenschaft in der Wissensgesellschaft. Opladen: Westdeutscher Verlag.

Hetmanek, A., Knogler, M., \& CHU Research Group (2018). Kritisches Denken als Unterrichtsziel: Von der Definition zur Förderung. www.clearinghouse-unterricht.de, Kurzreview 18. https://www.clearinghouse.edu.tum.de/reviews/lehrstrategienim-vergleich/kritisches-denken-als-unterrichtsziel-von-derdefinition-zur-foerderung/. Zugegriffen: 17. Mai 2019.

Jaeggi, R., \& Wesche, T. (2009). Einführung: Was ist Kritik? In R. Jaeggi \& T. Wesche (Hrsg.), Was ist Kritik? (S. 7-20). Frankfurt a. M.: Suhrkamp.

Jiménez-Aleixandre, M.P., \& Puig, B. (2012). Argumentation, evidence evaluation and critical thinking. In B. Fraser, K. Tobin \& C. McRobbie (Hrsg.), Second international handbook for science education, part two (S. 1001-1017). Dordrecht: Springer.

Kadir, A. M. A. (2017). What teacher knowledge matters in effectively developing critical thinkers in 21 st century curriculum? Thinking Skills and Creativity, 23, 79-90.

Kanfer, F. H., Reinecker, H., \& Schmelzer, D. (2012). Selbstmanagement-Therapie: Ein Lehrbuch für die klinische Praxis (5. Aufl.). Berlin, Heidelberg: Springer.

Kennedy, M., Fisher, M. B., \& Ennis, R.H. (1990). Critical thinking: literature review and needed research. In L. Idol \& B.F. Jones (Hrsg.), Educational values and cognitive instruction: implications for reform (S. 11-40). London: Routledge.

Klosterman, M.L., \& Sadler, T.D. (2010). Multi-level assessment of scientific content knowledge gains associated with socioscientific issues based instruction. International Journal of Science Education, 32, 1017-1043.

Kolst $\varnothing$, S. D., Bungum, B., Arnesen, E., Isnes, A., Kristensen, T., Mathiassen, K., et al. (2006). Science students' critical examination of scientific information related to socioscientific issues. Science Education, 90(4), 632-655.

Kuhn, D. (1999). A Developmental Model of Critical Thinking. Educational Researcher, 28(2), 16-26, 46. 
Lederman, N. G., Abd-El-Khalick, F., Bell, R., \& Schwartz, R. (2002). Views of nature of science questionnaire: toward valid and meaningful assessment of learner' conceptions of nature of science. Journal of Research in Science Teaching, 39, 497-521.

Lembeck, K.-H. (1996). Kritizismus. In P. Prechtl \& F.-P. Burkard (Hrsg.), Metzler-Philosophie-Lexikon (S. 279). Stuttgart: Metzler.

Lipman, M. (1988). Critical thinking-what Can it be? Educational Leadership, 46, 38-43.

McComas, W.F., \& Olson, J.K. (1998). The nature of science in international science education standards documents. In W.F. McComas (Hrsg.), The nature of science in science education: rationales and strategies (S. 41-52). Dordrecht: Kluwer Academic Publishers.

McMillan, J.H. (1987). Enhancing college students' critical thinking: a review of studies. Research in Higher Education, 26, 3-29.

McPeck, J.E. (1981). Critical thinking and education. New York: St. Martin's Press.

Moore, T. (2013). Critical thinking: seven definitions in search of a concept. Studies in Higher Education, 38(4), 506-522.

Neumann, I., \& Kremer, K. (2013). Nature of Science und epistemologische Überzeugungen - Ähnlichkeiten und Unterschiede. Zeitschrift für Didaktik der Naturwissenschaften, 19, 209-232.

OECD (2018). PISA 2015 Results in Focus. https://www.oecd.org/pisa/ pisa-2015-results-in-focus.pdf. Zugegriffen: 17. Mai 2019.

Osborne, J. (2014). Teaching critical thinking? New directions in science education. School Science Review, 95(352), 53-62.

Paul, R. W., \& Elder, L. (2014). Critical thinking: tools for taking charge of your professional and personal life. Upper Saddle River: Pearson Education.

Pithers, R. T., \& Soden, R. (2000). Critical thinking in education: a review. Educational Research, 42(3), 237-249.

Popper, K. R. (1984). Logik der Forschung. Tübingen: Mohr.

Rafolt, S., \& Kapelari, S. (2018). A Pilot Study for Promoting Students' Critical Thinking Through an Upper-Secondary Biology Class in Austria. Conference Proceedings: New Perspectives in Science Education (S. 487-491). Padova: libreriauniversitaria.it edizioni.

Rafolt, S., Kapelari, S., \& Kremer, K. (2018). Critical thinking in German-speaking biology curricula of Austria, Germany, Italy and Switzerland. In O. Finlayson, E. McLoughlin, S. Erduran \& P. Childs (Hrsg.), E-Book Proceedings of the ESERA 2017 Conference: Research, practice and collaboration in science education, Part 7 (S. 980-989). Dublin, Ireland: European Science Education Research Association.

Röttgers, K. (1975). Kritik und Praxis: Zur Geschichte des Kritikbegriffs von Kant bis Marx. Berlin: De Gruyter.

Sadler, T.D., \& Zeidler, D.L. (2005). Patterns of informal reasoning in the context of socioscientific decision making. Journal of Research in Science Teaching, 42, 112-138.

Schneiders, W. (1985). Vernünftiger Zweifel und wahre Eklektik: Zur Entstehung des modernen Kritikbegriffes. Studia Leibnitiana, 17(2), 143-161.

Schraw, G., Crippen, K. J., \& Hartley, K. (2006). Promoting self-regulation in science education: metacognition as part of a broader perspective on learning. Research in Science Education, 36, 111-139.
Sekretariat der Ständigen Konferenz der Kultusminister der Länder in der Bundesrepublik Deutschland (2005). Beschlüsse der Kultusministerkonferenz: Bildungsstandards im Fach Biologie für den Mittleren Schulabschluss. München, Neuwied: Luchterhand.

Siegel, H. (1988). Educating reason: rationality, critical thinking, and education. New York: Routledge.

Sieroka, N., Otto, V.I., \& Fokers, G. (2018). Gast-Editorial: Kritisches Denken in Lehre und Forschung - warum und wie? Angewandte Chemie, 130(51), 16812-16813.

Sinatra, G. M., Kienhues, D., \& Hofer, B. K. (2014). Addressing challenges to public understanding of science: epistemic cognition, motivated reasoning, and conceptual change. Educational Psychologist, 49(2), 123-138.

Stanovich, K.E. (2016). The comprehensive assessment of rational thinking. Educational Psychologist, 51, 23-34.

Tiruneh, D. T., De Cock, M., Weldeslassie, A. G., Elen, J., \& Janssen, R. (2017). Measuring critical thinking in physics: development and validation of a critical thinking test in electricity and magnetism. International Journal of Science and Mathematics Education, 15(4), 663-682.

Vieira, R.M., Tenreiro-Vieira, C., \& Martins, I.P. (2011). Critical thinking: conceptual clarification and its importance in science education. Science Education International, 2, 43-54.

Viennot, L., \& Décamp, N. (2016). Conceptual and critical development in student teachers: first steps toward an integrated compehension of osmosis. International Journal of Science Education, 38(14), 2197-2219.

Winch, C. (2003). Die Entwicklung kritischer Rationalität als pragmatische Aufgabe der Erziehung. Zeitschrift für Pädagogik, 49, 13-32.

Yacoubian, H.A. (2015). A framework for guiding future citizens to think critically about nature of science and socioscientific issues. Canadian Journal of Science, Mathematics and Technology Education, 15(3), 248-260.

Zeidler, D.L. (2014). Socioscientific issues as a curriculum emphasis. Theory, research, and practice. In N.G. Lederman \& S. K. Abell (Hrsg.), Handbook of research in science education (Bd. 2, S. 679-726). New York: Routledge.

Zeidler, D.L., \& Nichols, B.H. (2009). Socioscientific issues: theory and practice. Journal of Elementary Science Education, 21(2), 49-58.

Zeidler, D.L., Lederman, N. G., \& Taylor, S.C. (1992). Fallacies and student discourse: conceptualizing the role of critical thinking in science education. Science Education, 76(4), 437-450.

Zeidler, D. L., Sadler, T.D., Applebaum, S., \& Callahan, B.E. (2009). Advancing reflective judgment through socioscientific issues. Journal of Research in Science Teaching, 46, 74-101.

Zeidler, D. L., Sadler, T.D., Simmons, M.L., \& Howes, E. V. (2005). Beyond STS: a research-based framework for socioscientific issues education. Science Education, 89(3), 357-377.

Zeidler, D. L., Walker, K. A., Ackett, W. A., \& Simmons, M. L. (2002) Tangled up in views: beliefs in the nature of science and responses to socioscientific dilemmas. Science Education, 86(3), 343-367. 